\title{
Comparison of an Interstitial Cystitis/Bladder Pain Syndrome Clinical Cohort With Symptomatic Community Women From the RAND Interstitial Cystitis Epidemiology Study
}

\author{
Katy S. Konkle, Sandra H. Berry ${ }^{\star}$, Marc N. Elliott, Lara Hilton, Marika J. Suttorp, Daniel J. \\ Clauw $^{\dagger}$, and J. Quentin Clemens ${ }^{\ddagger}, \S$ \\ University of Michigan Medical Center, Ann Arbor, Michigan (KSK, DJC, JQC) and the RAND \\ Corporation, Santa Monica, California (SHB, MNE, LH, MJS)
}

\begin{abstract}
Purpose-The RAND Interstitial Cystitis Epidemiology survey estimated that $2.7 \%$ to $6.5 \%$ of United States women have urinary symptoms consistent with a diagnosis of interstitial cystitis/ bladder pain syndrome. We describe the demographic and clinical characteristics of the symptomatic community based RAND Interstitial Cystitis Epidemiology cohort, and compare them with those of a clinically based interstitial cystitis/bladder pain syndrome cohort.
\end{abstract}

\begin{abstract}
Materials and Methods-Subjects included 3,397 community women who met the criteria for the RAND Interstitial Cystitis Epidemiology high sensitivity case definition, and 277 women with an interstitial cystitis/bladder pain syndrome diagnosis recruited from specialist practices across the United States (clinical cohort). Questions focused on demographic information, symptom severity, quality of life indicators, concomitant diagnoses and treatment.
\end{abstract}

Results-Average symptom duration for both groups was approximately 14 years. Women in the clinical cohort reported worse baseline pain and maximum pain, although the absolute differences were small. Mean Interstitial Cystitis Symptom Index scores were approximately 11 for both groups, but mean Interstitial Cystitis Problem Index scores were 9.9 and 13.2 for the clinical cohort and the RAND Interstitial Cystitis Epidemiology cohort, respectively ( $\mathrm{p}<0.001$ ). The RAND Interstitial Cystitis Epidemiology subjects were more likely to be uninsured.

Conclusions-The RAND Interstitial Cystitis Epidemiology community cohort was remarkably similar to an interstitial cystitis/bladder pain syndrome clinical cohort with respect to demographics, symptoms and quality of life measures. In contrast to other chronic pain conditions for which clinical cohorts typically report worse symptoms and functional status than population based samples, our data suggest that many measures of symptom severity and functional impact are similar, and sometimes worse, in the RAND Interstitial Cystitis Epidemiology cohort. These findings suggest that interstitial cystitis/bladder pain syndrome is significantly burdensome, and likely to be underdiagnosed and undertreated in the United States.

\footnotetext{
*Financial interest and/or other relationship with RAND Corporation (Health Unit).

${ }^{\dagger}$ Financial interest and/or other relationship with Cypress Biosciences Inc., Eli Lilly and Company, Forest Laboratories Inc., Jazz Pharmaceuticals Inc., Merck \& Co. Inc, Pierre Fabre Pharmaceuticals USA, Pfizer Inc. and UCB Inc.

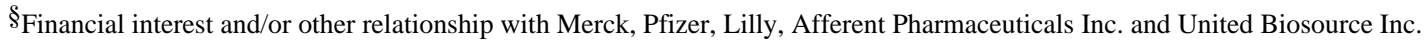
(C) 2012 by American Urological Association Education and Research, Inc.

${ }^{\ddagger}$ Correspondence: University of Michigan Medical Center, 3875 Taubman Center, 1500 E. Medical Center Dr., Ann Arbor, Michigan 48109-5330 (telephone: 734-615-1262; FAX: 734-615-9127; qclemens@umich.edu).
} 


\section{Keywords}

cystitis; interstitial; epidemiology; prevalence; questionnaires

Interstitial cystitis/bladder pain syndrome is an elusive disease that is difficult to define, diagnose and treat. Previous studies have suggested that the condition is more common than current rates of diagnosis would imply. ${ }^{1-5}$ We recently published national IC/BPS prevalence estimates as part of the RICE survey. ${ }^{6}$ Using standard case definitions with known sensitivity and specificity values, we estimated that between $2.7 \%$ and $6.5 \%$ of American women have bladder symptoms consistent with a diagnosis of IC/BPS. This suggests that IC/BPS is a pervasive condition affecting millions of American women.

In other chronic pain states such as fibromyalgia and irritable bowel syndrome it has been shown that clinical cohorts experience substantially greater symptom severity and quality of life impact than corresponding community samples. ${ }^{7-12}$ This referral and selection bias suggests that one cannot assess the comprehensive symptom burden of these conditions simply by extrapolating the symptom burden in the clinic by the known prevalence from population based studies. To ascertain if this pattern also persists in IC/BPS study populations, we examined the demographic and clinical characteristics of the large community based RICE cohort, and compared them with those of a smaller, clinically managed IC/BPS cohort typical of study populations traditionally used in IC/BPS research.

\section{METHODS}

\section{Clinical Cohort}

Details about recruitment of the clinical cohort have been previously published. ${ }^{13}$ This cohort consisted of 277 adult women previously diagnosed with IC/BPS with or without additional diagnoses such as endometriosis and vulvodynia. They were referred for RICE study participation by 8 urologists and 16 gynecologists across the United States with recognized expertise in managing IC/BPS. These women completed a questionnaire which included demographic information and information about IC/BPS symptoms.

\section{RICE High Sensitivity and High Specificity IC/BPS Case Definitions}

Using methods previously described 2 epidemiological case definitions for IC/BPS were developed. ${ }^{13}$ The high sensitivity definition yielded $81 \%$ sensitivity and $54 \%$ specificity, while the high specificity definition yielded $48 \%$ sensitivity and $83 \%$ specificity. These 2 IC/BPS case definitions were then used in a national survey designed to estimate the prevalence of IC/BPS in American women.

A 2-stage national screening process was then conducted using an ongoing survey mechanism. ${ }^{6}$ A total of 146,231 United States households were screened by telephone to identify adult women with IC/BPS symptoms. Previous diagnoses of similar disorders such as endometriosis or vulvodynia were not exclusionary. The survey identified 3,397 women who met the RICE high sensitivity definition, of whom 1,469 also met the RICE high specificity definition. For the purposes of this study those women who only met the criteria for the high sensitivity definition (but not the high specificity definition) were designated the RICE I cohort $(1,928)$, while the women who met the criteria for the RICE high sensitivity and high specificity definitions were designated the RICE II cohort $(1,469)$. All RICE women were used for comparative analysis against the clinical cohort. 


\section{Strategy for Comparative Analysis}

The questionnaires used in the original clinical cohort and RICE surveys contained many identical items measuring demographic and socioeconomic information, as well as symptom characteristics and medical management (diagnoses and treatment). In particular, both surveys included standardized, validated tools such as the ICSI,${ }^{14}$ ICPI ${ }^{14}$ and the SF- $36 .{ }^{15}$ These common items allowed us to compare responses from the RICE I and RICE II cohorts to those of the clinical cohort.

\section{Statistical Analysis}

For each of the 3 cohorts (RICE I, RICE II and clinical) descriptive statistics were calculated for the clinical characteristic variables of age, race/ethnicity, work loss, insurance status, employment status, social characteristics, symptom duration and severity, diagnostic tests, medication use, concomitant diagnoses, ICSI, ICPI and SF-36 scales. CC data were unweighted, and RICE data were weighted for sample design and nonresponse in all analyses. Analyses were conducted in SAS@ 9.2 using survey procedures to adjust for the effects of weighting RICE data. Differences between groups were tested using weighted linear and logistic regression for continuous and categorical variables, respectively. All reported sample sizes represent unweighted sample sizes whereas all reported percentages and means represent weighted results (where applicable). All SF-36 T-score means and ranges were adjusted for age and gender (mean 50, SD 10).

\section{RESULTS}

\section{Clinical Cohort vs Entire RICE Sample}

Demographics-Results for the comparison of the CC with the entire RICE cohort are presented in the table. In the 2 groups mean age (approximately 45 years) and employment rates were similar (36\% to 39\% employed full-time, $13 \%$ to $17 \%$ employed part-time and approximately $47 \%$ not employed, no significance). The RICE cohort included fewer white subjects ( $\mathrm{p}<0.01)$ and more Hispanic women $(10.8 \%$ vs $3.6 \%$, p <0.001). RICE women were considerably more likely to be uninsured $(13.4 \%$ vs $2.2 \%, \mathrm{p}<0.001)$. On average, RICE women were less likely to be married $(\mathrm{p}<0.001)$ and had more children $(\mathrm{p}<0.001)$.

Symptom severity-CC and RICE women reported similar bladder symptom duration (13.6 vs 14.6 years, respectively, no significance). CC women reported somewhat worse levels of baseline pain (5.9 vs 5.4, p <0.001) and maximum pain (8.0 vs 6.6, p <0.001). CC women scored slightly higher on the ICSI $(11.3$ vs $10.7, \mathrm{p}<0.05)$ and lower on the pain component of the SF-36 (38.3 vs 40.7, p <0.01, data not shown), indicating mildly worse symptom severity than the combined RICE cohort.

Quality of life/impact-Symptoms seem to have had a greater impact on employment in the clinical cohort. When asked, "Have bladder problems or pelvic pain kept you from working, or kept you from working full time?" $13.0 \%$ of employed RICE women and 35.4\% of employed CC women answered in the affirmative ( $\mathrm{p}<0.001)$. Although SF-36 T-score results suggest that RICE women had slightly better physical functional health than CC women (Physical 40.9 vs 38.7, p <0.05; Mental 45.1 vs 44.9, no significance), RICE women reported being significantly more bothered by their bladder symptoms as indicated by higher ICPI scores (13.2 vs 9.9, p <0.001).

Diagnosis/treatment-As expected, CC women had undergone far more IC/BPS diagnostic tests $(\mathrm{p}<0.001)$ and received many more condition specific treatments for their symptoms $(\mathrm{p}<0.001)$. Similar proportions of RICE and CC women were diagnosed with 
overactive bladder ( $29.5 \%$ vs $24.8 \%$, no significance) as was the case for treatment with medications for incontinence or urinary urgency. $\mathrm{CC}$ women were more likely to be diagnosed with fibromyalgia, chronic fatigue syndrome, irritable bowel syndrome, vulvodynia and endometriosis $(\mathrm{p}<0.001)$. Conversely, RICE women reported a higher rate of panic attacks ( $23.4 \%$ vs $14.1 \%, \mathrm{p}<0.001)$, although they were less likely to receive medications for anxiety $(19.9 \%$ vs $42.2 \%, \mathrm{p}<0.001)$.

\section{RICE I vs RICE II Sample}

Results for the comparison between the RICE I and RICE II cohorts are also presented in the table. Demographic characteristics for these 2 RICE subgroups were generally similar. The RICE I cohort included a lower proportion of white subjects, ( $p<0.001)$ and more black ( $p$ $<0.01)$ and Hispanic subjects $(7.4 \%$ vs $13.1 \%, \mathrm{p}<0.001)$ than the RICE II cohort.

Mean symptom duration and baseline pain scores were nearly identical for the 2 groups (14.6 years and 5.4 on a 10-point scale, respectively, no significance). RICE II cohort women reported slightly more severe maximum pain ( 6.8 vs $6.5, \mathrm{p}<0.001)$ and higher ICSI scores (11.5 vs 10.1, $\mathrm{p}<0.001)$. RICE II women experienced more work loss due to bladder symptoms $(15.0 \%$ vs $11.6 \%, \mathrm{p}<0.05)$, and had somewhat higher (more severe) ICPI scores $(14.2$ vs $12.5, \mathrm{p}<0.001)$ and slightly lower (worse) SF-36 T-scores (Physical 39.3 vs 42.1, p $<0.001$; Mental 44.8 vs 45.3 , no significance). Diagnostic and therapeutic interventions specific for IC/BPS were similarly infrequent in the RICE I and RICE II cohorts, except pentosan polysulfate, which was used more frequently in the RICE II cohort (3.3\% vs $1.1 \%$, $\mathrm{p}<0.01$ ). The rate of concomitant diagnoses was also similar, with small but statistically significant differences in fibromyalgia $(\mathrm{p}<0.05)$, irritable bowel syndrome $(\mathrm{p}<0.001)$ and overactive bladder $(\mathrm{p}<0.05)$. Both groups had received more medications for incontinence or urgency than for IC/BPS.

\section{DISCUSSION}

Our previous findings indicate that $6.5 \%$ of American women meet the RICE I (high sensitivity) IC/BPS criteria and $2.7 \%$ meet RICE II (high specificity) IC/BPS criteria, indicating that painful bladder symptoms are common in the community. ${ }^{6}$ In this study we compared basic clinical and demographic characteristics of all women meeting RICE I or II criteria with those of a previously described IC/BPS clinical cohort. Although some differences were observed among these clinical and community groups, overall these IC/ BPS samples were strikingly similar.

The most significant demographic difference observed between the 2 groups was the lower rate of health insurance in the RICE cohort $(2.2 \%$ vs $13.4 \%, \mathrm{p}<0.001)$, a predictable consequence of clinical recruitment. By definition, the $\mathrm{CC}$ women were receiving treatment from a physician and would be expected to have insurance coverage for these services. Other differences in age, marital status and race/ethnicity may have reflected differences in insurance status or other aspects of access to health care.

Both populations described, on average, approximately 14 years of bladder symptoms. CC women reported slightly worse symptom severity, although the actual differences in scores were small and of questionable clinical significance. The burden of these symptoms was reflected by ICPI and SF-36 scores, as well as extensive work loss due to bladder problems or pelvic pain. Of note, ICPI scores suggested that RICE women were significantly more bothered by bladder symptoms than CC women, despite slightly less symptom severity. A possible explanation for this paradox is that access to care for IC/BPS symptoms may provide psychological or coping benefits which could reduce the impact of the symptoms. 
Importantly $13 \%$ of RICE women and more than a third of all CC women reported that bladder problems or pelvic pain kept them from working or kept them from working fulltime. It is unclear why $\mathrm{CC}$ women were more than twice as likely to have been unable to work compared to women in the community. However, the interpretation of being unable to work is individual and higher rates of being married may reduce the need to work. As discussed, CC women did consistently report slightly worse symptom severity, although the differences were small and do not fully explain this observation.

As expected from a comparison of clinical and community cohorts, large differences were seen between the 2 groups in terms of diagnoses and treatment. We did not compare the frequency of IC/BPS specific diagnoses because this was an inclusion criterion for the CC, and so differed by definition. The finding that $\mathrm{CC}$ women were more likely to be diagnosed with nearly any of the concomitant diagnoses commonly present in patients with IC/BPS (eg fibromyalgia, irritable bowel syndrome, depression etc) was not surprising. Such differences are likely to reflect greater access to and increased interactions with the health care system, factors inherent in a sample receiving specialist care. While precluded in this instance by limited interview time, future work might also address the presence of concomitant nonbladder symptoms (rather than diagnoses). Any such differences would almost entirely reflect differences in access and, thus, might provide context for differences in rates of diagnosis for IC/BPS associated conditions.

Although these clinical and community based women have nearly identical bladder complaints, it appears that when they present for medical care they are treated differently. Given that all of the CC women had previously been diagnosed with IC/BPS and that $98 \%$ reported having had some form of health insurance, we anticipated and observed higher levels of diagnostic tests and disease specific treatment in this group. However, CC and RICE women were diagnosed with and treated for overactive bladder at near equal rates, suggesting that RICE women do seek and receive some form of care for bladder symptoms, but often do not receive IC/BPS specific care. We speculate that these treatment discrepancies likely reflect differences in diagnoses and care provided by specialists (eg urologists or gynecologists) vs primary care providers.

Previous studies of chronic pain populations have shown that clinical cohorts have substantially greater symptom severity and disease burden than community based samples. ${ }^{7-12}$ These findings have been interpreted to suggest that large scale population based studies tend to identify trivial symptoms which cause minimal bother. Our findings are strikingly different and suggest that the community prevalence rates identified in the RICE survey do, in fact, identify women with real problems who are significantly bothered by their symptoms.

Furthermore, our comparison of the high sensitivity and high specificity RICE cohorts demonstrated substantial similarity in demographics, symptom severity, diagnoses, treatments and quality of life scores. Observed differences between the 2 RICE cohorts were small and we believe unlikely to be relevant in the setting of real medical management. Of note, similar epidemiological studies conducted for fibromyalgia and irritable bowel syndrome revealed few clinically meaningful differences between high sensitivity and high specificity populations. ${ }^{16,17}$

\section{CONCLUSIONS}

Results of the RICE prevalence study indicate that between 3.3 and 7.9 million American women older than age 18 years have bladder symptoms associated with IC/BPS. Unlike other chronic pain populations, our comparison of these RICE diagnosed women with a 
clinical cohort demonstrates striking demographic, symptomatic and quality of life similarities. These findings strengthen the existing body of evidence which suggests that this condition is substantially burdensome, and likely underdiagnosed and undertreated in the United States.

\section{Acknowledgments}

Study received institutional review board approval (RAND IRB \# b4822-04-01).

Supported by National Institute of Diabetes and Digestive and Kidney Diseases Grant UO1 DK070234.

\section{Abbreviations and Acronyms}

$\begin{array}{ll}\text { CC } & \text { clinical cohort } \\ \text { IC/BPS } & \text { interstitial cystitis/bladder pain syndrome } \\ \text { ICPI } & \text { Interstitial Cystitis Problem Index } \\ \text { ICSI } & \text { Interstitial Cystitis Symptom Index } \\ \text { RICE } & \text { RAND Interstitial Cystitis Epidemiology } \\ \text { RICE I } & \text { RICE high sensitivity } \\ \text { RICE II } & \text { RICE high specificity } \\ \text { SF-36 }{ }^{\circledR} & \text { short form health survey }\end{array}$

\section{REFERENCES}

1. Clemens JQ, Meenan RT, O'Keeffe Rosetti MC, et al. Prevalence of interstitial cystitis symptoms in a managed care population. J Urol. 2005; 174:576. [PubMed: 16006901]

2. Clemens JQ, Link CL, Eggers PW, et al. Prevalence of painful bladder symptoms and effect on quality of life in black, Hispanic and white men and women. J Urol. 2007; 177:1390. [PubMed: 17382739]

3. Leppilahti M, Tammela TL, Huhtala H, et al. Prevalence of symptoms related to interstitial cystitis in women: a population based study in Finland. J Urol. 2002; 168:139. [PubMed: 12050508]

4. Lifford KL, Curhan GC. Prevalence of painful bladder syndrome in older women. J Urol. 2009; 73:494.

5. Curhan GC, Speizer FE, Hunter DJ, et al. Epidemiology of interstitial cystitis: a population based study. J Urol. 1999; 161:549. [PubMed: 9915446]

6. Berry SH, Elliott MN, Suttorp M, et al. Prevalence of symptoms of bladder pain syndrome/ interstitial cystitis among adult females in the United States. J Urol. 2011; 186:540. [PubMed: 21683389]

7. Drossman DA, McKee DC, Sandler RS, et al. Psychosocial factors in the irritable bowel syndrome. A multivariate study of patients and nonpatients with irritable bowel syndrome. Gastroenterology. 1988; 95:701. [PubMed: 3396817]

8. Roy R, Thomas M, Berger S. A comparative study of Canadian nonclinical and British pain clinic subjects. Clin J Pain. 1990; 6:276. [PubMed: 2135027]

9. Heaton KW, Ghosh S, Braddon FE. How bad are the symptoms and bowel dysfunction of patients with the irritable bowel syndrome? A prospective, controlled study with emphasis on stool form. Gut. 1991; 32:73. [PubMed: 1991641]

10. Sperber AD, Carmel S, Atzmon Y, et al. Use of the Functional Bowel Disorder Severity Index (FBDSI) in a study of patients with the irritable bowel syndrome and fibromyalgia. Am J Gastroenterol. 2000; 95:995. [PubMed: 10763949]

11. Kersh BC, Bradley LA, Alarcon GS, et al. Psychosocial and health status variables independently predict health care seeking in fibromyalgia. Arthritis Rheum. 2001; 45:362. [PubMed: 11501724] 
12. Rode $\mathrm{S}$, Salkovskis $\mathrm{P}$, Dowd $\mathrm{H}$, et al. Health anxiety levels in chronic pain clinic attenders. J Psychosom Res. 2006; 60:155. [PubMed: 16439268]

13. Berry SH, Bogart LM, Pham C, et al. Development, validation and testing of an epidemiological case definition of interstitial cystitis/painful bladder syndrome. J Urol. 2010; 183:1848. [PubMed: 20303099]

14. O'Leary MP, Sant GR, Fowler FJ, et al. The interstitial cystitis symptom index and problem index. Urology. 1997; 49:58. [PubMed: 9146003]

15. Ware, JE.; Kosinski, M.; Gandek, B. SF-36 Health Survey: Manual and Interpretation Guide. Lincoln, Rhode Island: QualityMetric Inc.; 2005.

16. Arpino C, Carrieri MP, Valesini G, et al. Idiopathic chronic fatigue and chronic fatigue syndrome: a comparison of two case-definitions. Ann Ist Super Sanita. 1999; 35:435. [PubMed: 10721210]

17. Boyce PM, Koloski NA, Talley NJ. Irritable bowel syndrome according to varying diagnostic criteria: are the new Rome II criteria unnecessarily restrictive for research and practice? Am J Gastroenterol. 2000; 95:3176. [PubMed: 11095338] 


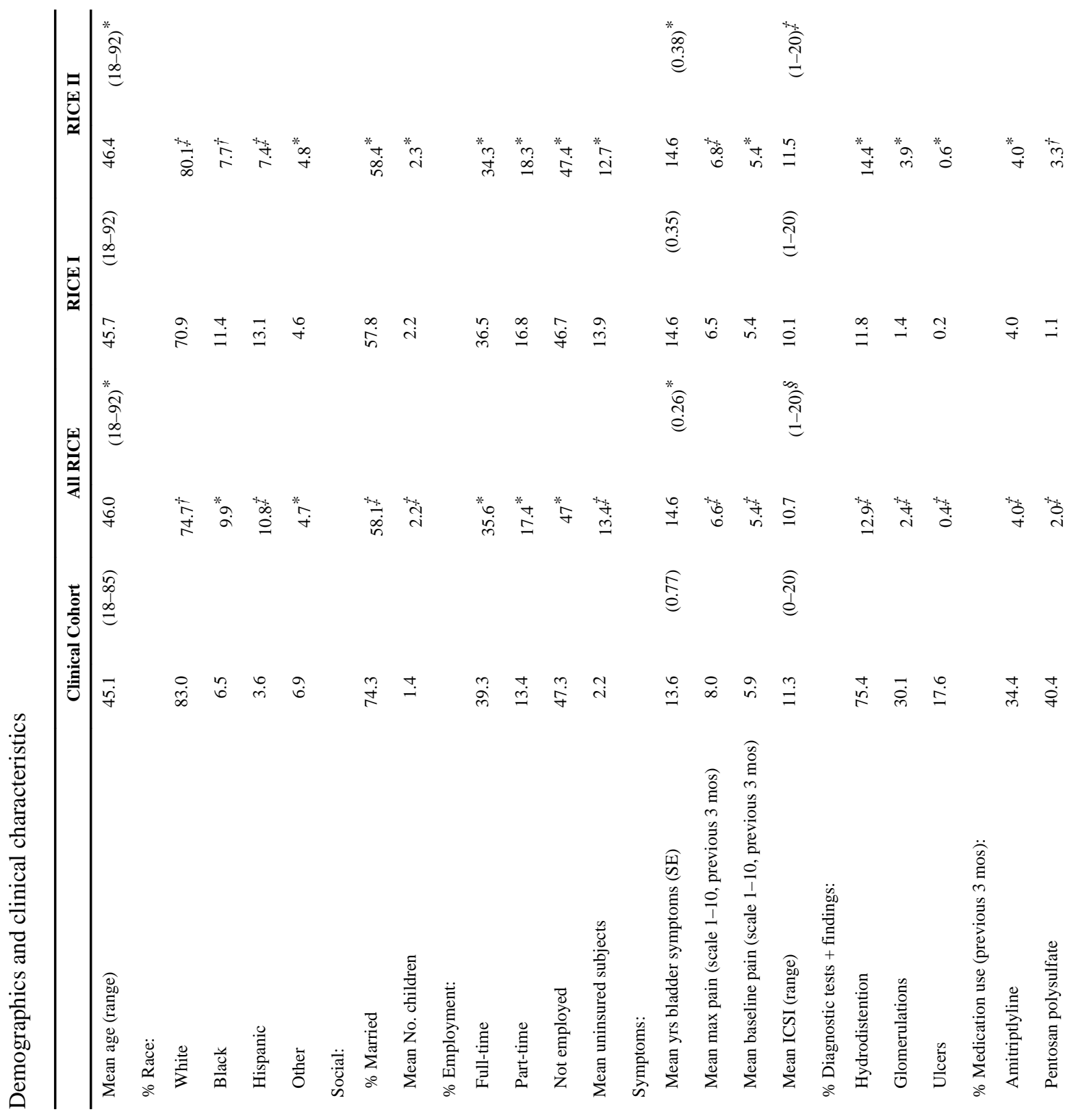




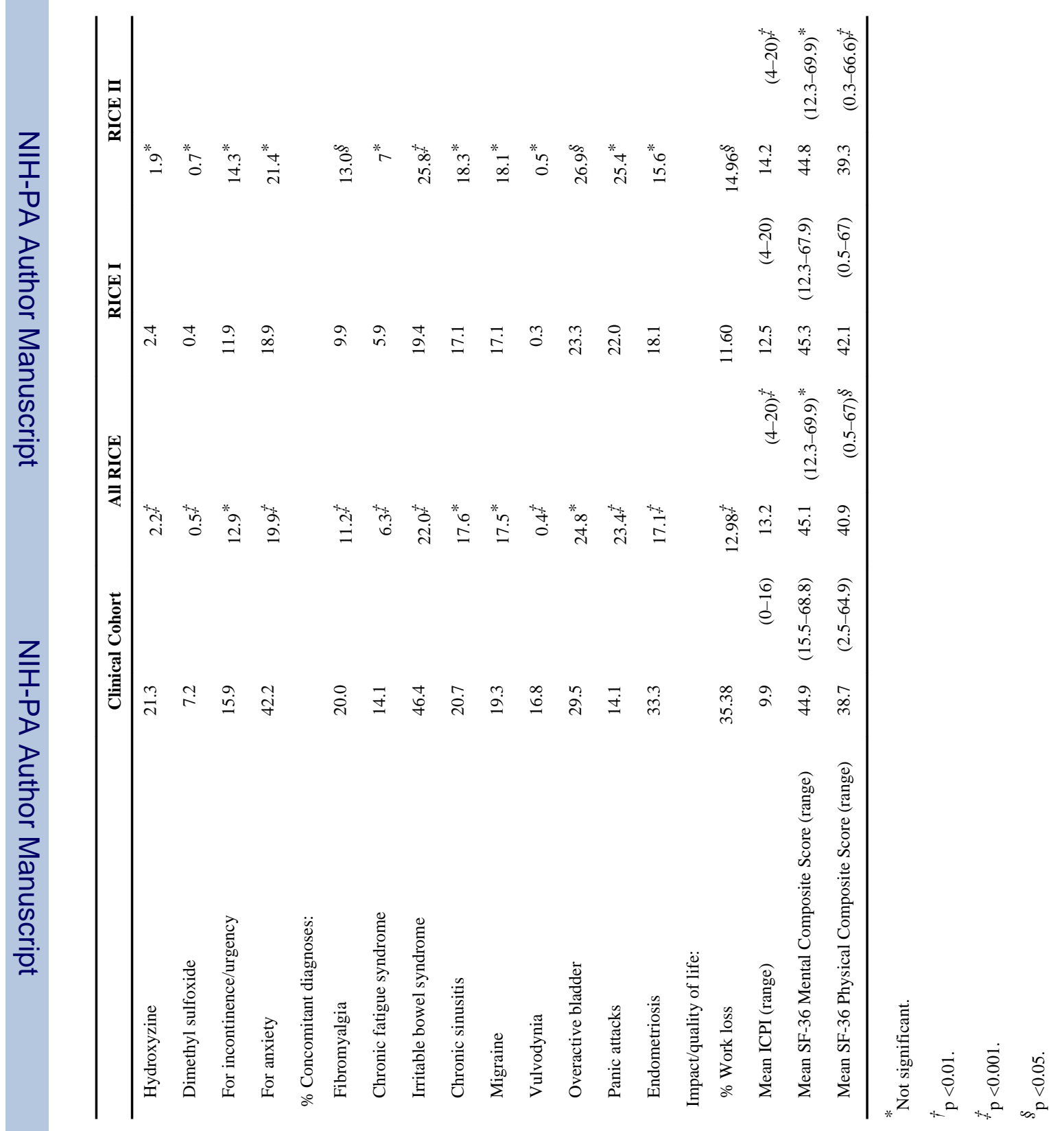

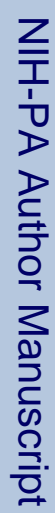

